\title{
Charm Production with Jets at H1
}

\author{
Sebastian Schmidt \\ Deutsches Elektronen-Synchrotron \\ Notkestrasse 85, 22607 Hamburg - Germany
}

\begin{abstract}
Measurements of inclusive $D^{* \pm}$ meson production in photoproduction and deep-inelastic scattering at HERA are presented. To gain a further understanding of the production mechanism of charm, events containing jets, in addition to the $D^{* \pm}$, are selected. This allows to investigate quantities sensitive to the partons interacting in the hard subprocess such as the fractional momentum w.r.t the photon and proton, and the azimuthal angle between the jets. The data are compared with theoretical models such as NLO pQCD calculations based on the DGLAP evolution scheme or those based on CCFM evolution and $k_{t}$-unintegrated gluon distributions.
\end{abstract}

\section{Introduction}

Charm is produced at HERA in deep-inelastic scattering (DIS) and in photoproduction predominantly by boson-gluon-fusion between a photon originating from the positron and a gluon originating from the proton. A full understanding of this interaction is only possible if the kinematic properties of the two outgoing hard partons are completely determined. The well known tagging of charm events by $D^{* \pm}$ mesons decaying in the so called "Golden Channel" results in high precision reconstruction of the kinematic quantities, but considerably limits the available event sample due to the low branching ratios. Instead of studying events with a double tagged charm one can approximate one or both of the partons by jets and thus gain deeper insight into the production mechanism of charm at HERA.

\section{Inclusive cross sections}

Recently measurements of inclusive $D^{* \pm}$ meson production in DIS [2] and photoproduction [3] have been performed at HERA with the H1 detector. The results are compared with different theoretical predictions based on NLO QCD using DGLAP evolution. Predictions in the fixed flavour number scheme (FFNS) are made using the HvQDIS[4] and FNMR[7] programs for DIS and photoproduction, respectively. Predictions using the zero mass variable flavour number scheme (ZM-VFNS) [6], in which the charm mass is neglected, are available in the DIS case. Predictions using the general mass variable flavour number scheme (GM-VFNS) [8], which combines aspects of the FFNS and ZMVFNS, are available for photoproduction. In addition, the results are compared with predictions based on CCFM evolution involving the $k_{t}$-unintegrated gluon distribution in the proton calculated using the CASCADE program [5].

The kinematic range of the DIS analysis is described by restrictions on the photon virtuality $2 \leq Q^{2} \leq 100 \mathrm{GeV}^{2}$ and the inelasticity $0.05 \leq y \leq 0.7$. For the photoproduction sample conditions on $Q^{2}<0.01 \mathrm{GeV}^{2}$ and on the photon-proton centre-of-mass energy $171<W_{\gamma p}<256 \mathrm{GeV}$ are applied.

The overall description of the DIS data by the available models is reasonable. Some deviations are observed in the forward region of large pseudorapidity $\eta$ which are also reflected in an excess of the data over the models in the correlated region of low inelasticity $z$ (see Fig. 1). 
The photoproduction data are described less well, especially if distributions are considered which are sensitive to the phase space of the outgoing charm quark $\left(p_{t}\right.$ and $\eta)$. In the $z$ distribution a similar excess of data over the models is observed as in the DIS case. In general for the
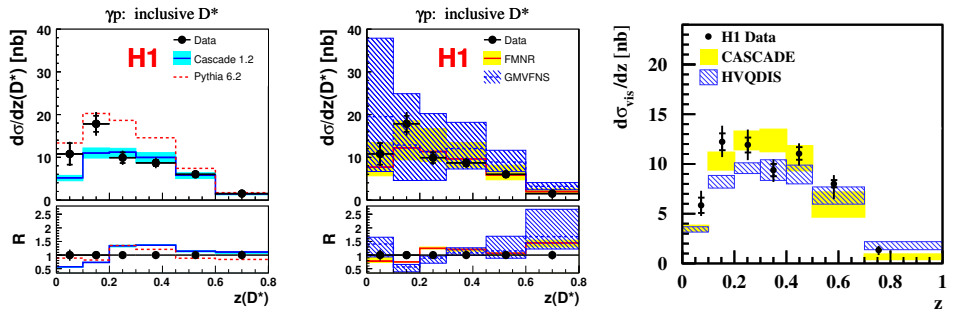

Figure 1: Differential distribution in bins of the inelasticity $z$ for inclusive $D^{* \pm}$ meson production in photoproduction (left and middle plot) and DIS (right plot).

photoproduction analysis - and to a lesser extent in the DIS case - the precision of the data is much higher than the accuracy of the NLO calculations, which is dominated by the uncertainty on the charm mass, the choice of factorisation and renormalisation scale and the parametrisation of the non-perturbative fragmentation.

\section{$3 \quad$ Production of $D^{* \pm}$ mesons with jets}

In order to define a sample of $D^{* \pm}$ mesons with jets the $k_{\perp}$-cluster algorithm is applied to hadronic final state objects. For events containing a $D^{* \pm}$ meson candidate these are reconstructed from tracks and calorimeter depositions in the Breit and laboratory frame for DIS and photoproduction, respectively. The four-vector of the reconstructed $D^{* \pm}$ meson is used instead of the fourvectors of its three decay particles. The leading jet is required to have a transverse energy of $E_{T}>4 \mathrm{GeV}$, the next-to-leading jet a transverse energy of $E_{T}>3 \mathrm{GeV}$.

In the following a selection of interesting jet observables are discussed.

\subsection{The observables $x_{\gamma}$ and $x_{g}$}

At LO the observables $x_{\gamma}^{\text {obs }}$ and $x_{g}^{\text {obs }}$ give the observed fraction of the photon momentum carried by the parton involved in the hard
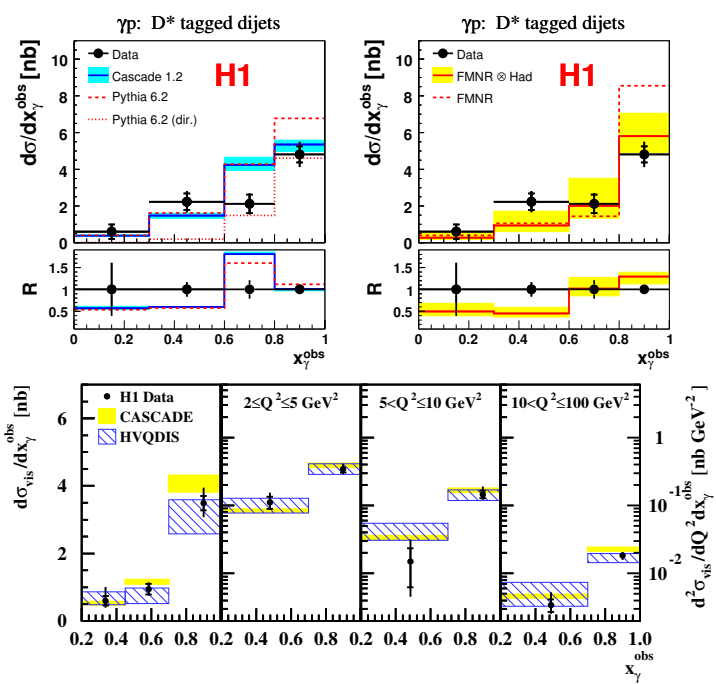

Figure 2: Differential distribution in bins of $x_{\gamma}$ for the photoproduction case (upper plots) and the DIS case (lower plot). For DIS also double differential distributions in bins of $x_{\gamma}^{\text {obs }}$ and $Q^{2}$ are shown. subprocess and the observed fraction of the proton momentum carried by the gluon, respectively. The determination of both quantities involves the reconstruction of the 4-vectors of the partons emerging from the hard subprocess, which are approximated by the two jets.

The distribution of $x_{\gamma}^{\text {obs }}$ (see Fig. 2) peaks for both the DIS and the photoproduction case at 1 as expected from direct processes. It has however significant contributions at lower values from so-called resolved processes. In the DIS case for HVQDIS there seems to be 
no need for an additional resolved contribution beyond what is already present at NLO. CASCADE provides also a reasonable description.

For photoproduction the large cross section at small $x_{\gamma}^{\text {obs }}$ shows that processes beyond direct photon-gluon-fusion are needed to describe the data in the collinear approach. This can be seen by comparison with the prediction for PYTHIA direct. Both Pythia and CASCADE give a poor description of the $x_{\gamma}^{\text {obs }}$ distribution. All predictions underestimate the region of low $x_{\gamma}^{\text {obs }}$ which can be seen in the normalised shape $R$.

Figure 3 shows differential cross sections

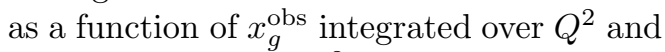
in three regions of $Q^{2}$ for the DIS sample. Both HvQdis and CASCADE describe the $Q^{2}$ dependence of $x_{g}^{\text {obs }}$ with the default parton density functions (PDF) CTEQ5F3 (for HVQDIS) and A0 (for CASCADE). The sensitivity to the PDFs has been investigated by comparing with the predictions of HvQDIS using the MRST2004F3NLO parametrisation and with the predictions of CASCADE using the parametrisation J2003 set-1 for the unintegrated gluon density. The differences in the cross section are small, compared to the large theoretical and statistical

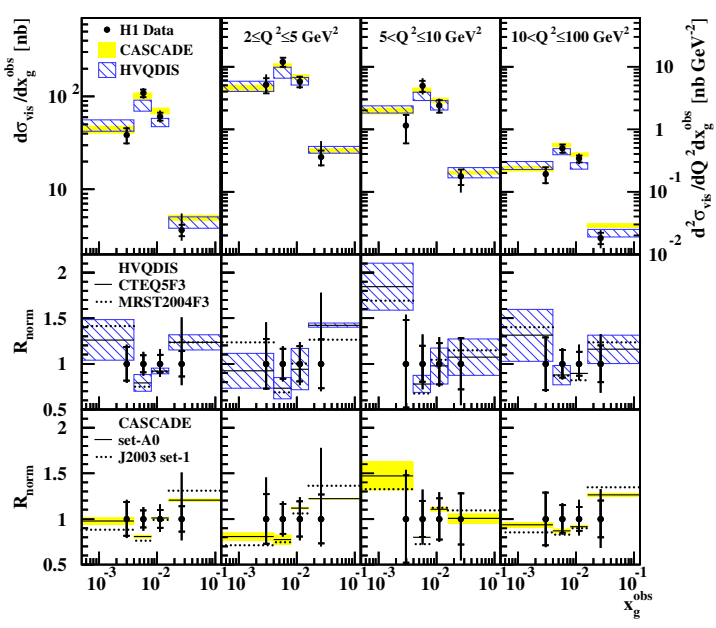

Figure 3: Differential distributions in bins of $x_{g}^{\text {obs }}$ for DIS. In addition double differential distributions in bins of $x_{g}^{\mathrm{obs}}$ and $Q^{2}$ are shown. In the lower part of the plot the normalised ratio $R_{\text {norm }}$ between data and the predictions is shown. Here an additional comparison between predictions using the default PDFs and alternative PDFs is performed. uncertainties.

\subsection{The observable $\Delta \phi$}

Another interesting aspect of the production process of charm at HERA is the distribution of the azimuthal angle between the two jets. ${ }^{a}$ In leading order this distribution consists of a delta peak at 180 degrees originating from back-to-back configurations in the transverse plane. All contributions away from this value are due to higher order (and resolution) effects. As for analysies of inclusive dijets at HERA[9] disagreement between data and the theory models is observed (see Fig. 4). In both kinematic regimes for the NLO-DGLAP approach (HVQDIS) higher order contributions at small angles are lacking. However, the model based on the CCFM evolution equation using unintegrated gluon densities (CASCADE) overestimates the contributions in that region. This is most probably due to a too broad unintegrated gluon density.

${ }^{a}$ In the analysis of the photoproduction sample for this observable instead of the two jets representing the charm and the anti-charm quark the $D^{* \pm}$ meson and a single jet not associated with the $D^{* \pm}$ meson are studied. 


\section{Summary}

In recent $\mathrm{H} 1$ analyses production cross sections of $D^{* \pm}$ mesons with jets have been studied in DIS as well as in photoproduction. They have been compared with theoretical predictions to achieve insight into the production mechanism of charm at HERA. The observable $x_{\gamma}$ allows to separate the composition of direct and resolved processes. With the help of the observable $x_{g}$ present parton density functions can be verified. The azimuthal angle between the jets ${ }^{\mathrm{b}}$ $\Delta \phi$ shows, depending on the model used, missing higher order contributions or a too broad $k_{t}$-unintegrated gluon density.

In general it is observed that the significance of the measurements is limited by about the same amount in the precision of the data and in the uncertainties of the theoretical calculations.

\section{References}

[1] Slides: http: //indico. cern. ch/contributionDisplay $\cdot$ py? contribId=192\&sessionId=5\&conf Id=9499

[2] H1 Collab., A. Aktas et al., Accepted by Eur. Phys. J. C [hep-ex/0701023v1] (2007).

[3] H1 Collab., A. Aktas et al., Accepted by Eur. Phys. J. C [hep-ex/0608042v1] (2006).

[4] B.W. Harris and J. Smith, Nucl. Phys. B 452 (1995) 109 [hep-ph/9503484]; B.W. Harris and J. Smith, Phys. Lett. B 353 (1995) 353 [Erratum-ibid. B 359 (1995) 423] [hep-ph/9502312].

[5] H. Jung, Comput. Phys. Commun. 143 (2002) 100 [hep-ph/0109102].

[6] B.A. Kniehl, G. Kramer and M. Maniatis, Nucl. Phys. B 771 (2005) 345; Erratum 720 (2005) 345 [hep-ph/0109102]; I. Schienbein, Procs. of Ringberg Workshop "New Trend in HEAR Physics 2005", Eds. G. Grindhammer et al., Ringberg Castle, Tegernsee, Germany, 2-7 Oct 2005, World Scientific Publishing Singapore 2006 [hep-ph/0601235].

[7] S. Frixione, P. Nason and G. Ridolfi, Nucl. Phys. B 454 (1995) 3 [hep-ph/9506226]; S. Frixione, M.L. Mangano, P. Nason and G. Ridolfi, Phys. Lett. B 348 (1995) 633 [hep-ph/9412348].

[8] B.A. Kniehl, G. Kramer, I. Schienbein and H. Spiesberger, Phys. Rev. D 71 (2005) 014018 [hepph/0410289]; B.A. Kniehl, G. Kramer, I. Schienbein and H. Spiesberger, Eur. Phys. J. C 41 (2005) 199 [hep-ph/0502194].

[9] H1 Collab., A. Aktas et al., Eur. Phys. J. C 33 (2004) 477 [hep-ex/0310019].

\footnotetext{
${ }^{\mathrm{b}}$ or between the $D^{* \pm}$ meson and a jet in the photoproduction case
} 